\title{
Secreted antigens of the amastigote and promastigote forms OF LEISHMANIA INFANTUM INDUCING A HUMORAL RESPONSE IN HUMANS AND DOGS
}

\author{
CIBREluS P.*, PRÉCIGOUT E.*, SERENO D.**, CARCY B.*, LEMESRE J.L.** \& GORENFLOT A.*
}

\begin{abstract}
Summary :
To study the antigens secreted by promastigote and amastigote forms of Leishmania infantum which are able to induce a humoral response in human patients and dogs, we have carried out immunoprecipitation assays with different supernatants of in vitro cultured parasites, metabolically labelled with [ ${ }^{35}$ S]methionine, using serum samples from human patients and dogs. In addition, some metabolic labelling experiments were performed daily during the in vitro culture parasite's life cycle to follow the time course excretion-secretion of parasitic antigens. The results demonstrated that the two different hosts developed an antibody response against secreted antigens of both stages of Leishmania infantum. Nevertheless, the humoral response directed against the excretedsecreted antigens of the promastigote forms was qualitatively and quantitatively different when we compare the human and the dog immune responses. On the other hand, when the excreted-secreted antigens of the amastigote forms are immunoprecipitated with either human or canine immune serum, the humoral response is similar. In addition, the time course study showed that excretionsecretion of antigens was qualitatively and quantitatively modulated during the parasitic in vitro life cycle.
\end{abstract}

KEY WORDS : Leishmania infantum, excreted-secreted antigens, humoral immunity, amastigote, promastigote.

ABBREVIATIONS : VL: visceral leishmaniasis; AMES-antigens: amastigote excreted-secreted antigens; PMES-antigens: promastigote excreted-secreted antigens.
Résumé : ANTIGÈNES SÉCRÉTÉS PAR LES FORMES AMASTIGOTES ET PROMASTIGOTES DE LEISHMANIA INFANTUM ET INDUISANT UNE RÉPONSE HUMORALE CHEZ L'HOMME ET LE CHIEN

Afin d'étudier les antigènes sécrétés par les formes promastigotes et amastigotes de Leishmania infantum capable d'induire une réponse humorale nous avons immunoprécipité différents surnageants de culture in vitro, marqués à la méthionine $\left[5^{35}\right], a ̀$ l'aide de sérums de malades ou de chiens. De plus, certains marquages métaboliques ont été effectués chaque jour et ceci durant un cycle complet de développement in vitro de L. infantum afin d'avoir un suivi temporel de l'excrétion-sécrétion des antigènes parasitaires. Les résultats obtenus ont montré que les deux différents hôtes développaient une réponse humorale contre les antigènes sécrétés par chacun des deux stades parasitaires de L. infantum. Néanmoins, la réponse humorale dirigée contre les antigènes d'excrétion-sécrétion du stade promastigote est qualitativement et quantitativement différente si l'on compare la réponse humorale humaine et canine. Par contre, que l'on immunoprécipite avec un sérum humain ou canin, la réponse humorale dirigée contre les antigènes d'excrétion-sécrétion du stade amastigote est similaire. De plus, l'étude temporelle a montré que l'excrétion-sécrétion des antigènes était qualitativement et quantitativement modulée au cours du cycle parasitaire in vitro de L. infantum.

MOTS CLÉS : Leishmania infantum, antigène d'excrétion-sécrétion, immunité humorale, forme amastigote, forme promastigote.

L. chagasi in the New World) are responsible for the visceral leishmaniasis which is characterized, in its active form, by a lack of impaired T-cell-mediated immune responses and a marked hypergammaglobulinaemia (Carvalho et al., 1981; Galvão-Castro et al., 1984). Leishmania presents with two morphologically distinct forms: $i$ ) the flagellated promastigote forms, transmitted to the vertebrate host by the bite of an infected sandfly, are found in the alimentary tract of the vector and, $i$ ) in the mammalian host, Leishmania replicates exclusively as the aflagellated amastigote form inside the macrophage where they induce strong changes in the cell functions and morphology of macrophages such as the formation of a phagolysosome or the down regulation of class II MHC (for a review see : Alexander \& Russell, 1992).

During its life cycle, Leishmania organisms secrete various antigens that could be directly implicated in many immunological or biochemical alterations of the

\footnotetext{
* EA MENRT 2413 : Immunothérapie et Chimiothérapie des Parasitoses et des Mycoses, Laboratoire de Biologie Cellulaire et Moléculaire, UFR des sciences Pharmaceutiques et Biologiques, 15, avenue Charles Flahault, 34060 Montpellier Cedex 1, France.

** Laboratoire de Biologie Parasitaire, ORSTOM, BP 5045, 34032 Montpellier Cedex 1, France.

Correspondence: André Gorenflot.

Tel : $33(0) 467546481$ - Fax : $33(0) 467546621$

E-mail: agorenflot@ww3.pharma.univ-montp1.fr
} 
host cell. Different glycoconjugates or glycoproteins such as lipophosphoglycan (Handman et al., 1984; King et al., 1987) or acid phosphatase (Bates et al., 1990; Ilg et al., 1991) have been shown to be secreted from the promastigote forms of Leishmania. Similarly, other studies have demonstrated $i$ ) the secretion of amastigote antigens into the phagolysosomal compartment via the flagellar pocket of the parasite (for review: Chakraborty \& Basu, 1997), ii) the presence of Leishmania-derived antigens on the surface of the parasitized macrophages (Berman \& Dwyer, 1981; Williams et al., 1986). Among all the described amastigote antigens of Leishmania species, some of them are well characterized: an acid phosphatase (Bates et al., 1989; Doyle \& Dwyer, 1993), a proteophosphoglycan, (Ilg et al., 1995), a sucrase (Blum \& Opperdoes, 1994), a glutathione binding $66 \mathrm{kDa}$ polypeptide (Yahiaoui et al., 1993) and a protective LACK antigen (Prina et al, 1996).

The recent development of axenic cultures of amastigote forms from different Leishmania species (Bates et al., 1992; Lemesre et al., 1997) have provided new opportunities to characterize the antigens excreted by the mammalian stage of the parasite. In this paper, we studied the humoral response of dogs and men against excreted-secreted antigens from Leishmania infantum amastigotes and promastigotes forms.

\section{MATERIALS AND METHODS}

\section{INFANTUM IN VITRO CULTURES}

The amastigote and promastigote forms of L. infantum MHOM/MA(BE)/67/ITMAP-263 clone 2 were used in the present study.

Axenically grown amastigotes were maintained at 37 $+/-1^{\circ} \mathrm{C}$ under $5 \% \mathrm{CO}_{2}$ atmosphere by subpassaging every five days in cell-free medium called MAA/20. MAA/20 consisted of modified medium 199 (Life Technologies, France) balanced with Hanks'salts, supplemented with $0.01 \mathrm{mM}$ bathocuproine disulfonic acid, $3 \mathrm{mM}$ L-cysteine, $5 \mathrm{mM}$ L-glutamine, $0.023 \mathrm{mM}$ hemin, $5 \mathrm{mM}$ D-glucose, $4 \mathrm{mM} \mathrm{NaHCO}_{3}, 0.5 \%$ soya tryptocasein and $25 \mathrm{mM}$ Hepes. The $\mathrm{pH}$ was adjusted at 6.5 and $20 \%$ heat-inactivated fetal calf serum (FCS) was added (Lemesre et al., 1997). The starting inoculum was $5 \times 10^{5}$ amastigotes $/ \mathrm{ml}$ and $10^{7}$ to $5 \times 10^{7}$ parasites were regularly obtained by day 5 .

Promastigote cultures were derived from axenically cultured amastigote stages and were maintained at $25+/-$ $1^{\circ} \mathrm{C}$ by weekly subculturing in medium RPMI 1640 (Life Technologies, France) buffered with 25 mM HEPES and $2 \mathrm{mM} \mathrm{NaHCO}_{3}, \mathrm{pH} 7.2$, supplemented with $10 \%$ heatinactived FCS. The starting inoculum was $5 \times 10^{5}$ promastigotes $/ \mathrm{ml}$.

\section{HUMAN AND DOG SERUM SAMPLES}

Human sera were obtained from five South-American patients infected with Leishmania chagasi (acute leishmaniasis), the causative agent of American visceral leishmaniasis (VL). The VL-infected human sera were: HIM-A1, HIM-A3, HIM-C1, HIM-V1 and HIM-L1. The VL-infected canine sera were obtained from four dogs (acute infection) living in the mediterranean endemic area and infected with Leishmania infantum. The canine sera were: CIS-14, CIS-15, CIS-16 and CIS-18. Sera from healthy subjects (human or dog) were used as negative controls.

\section{MetaBOLIC RADIOLABELLing}

Two different procedures of metabolic radiolabelling were performed. In a first experiment, the Leishmania parasites were labelled during a complete in vitro life cycle. When the in vitro cultures were initiated, $100 \mu \mathrm{Ci} / \mathrm{ml}$ of $\left.{ }^{35} \mathrm{~S}\right]$-methionine were added to $\mathrm{MAA} / 20$ medium and the labelled parasites were collected after five days for the amastigote forms and seven days for the promastigote forms. These labelled antigens were called "one cycle antigens".

In a second experiment, promastigotes and amastigotes were cultured in RPMI 1640 with $10 \%$ FCS and in MAA/20 with $20 \%$ FCS respectively, and supplemented with $\left.{ }^{35} \mathrm{~S}\right]$-methionine $(100 \mu \mathrm{Ci} / \mathrm{ml})$ for 24 hours. The same procedure was also utilized for the promastigote forms, except that it was carried out during seven days. On day 0 , five culture flasks were initiated with the amastigotes and seven with the promastigotes (one flask for each day of in vitro culture) and, for each parasitic form, $\left[{ }^{35} \mathrm{~S}\right]$-methionine was only added to one culture flask. On day 1, the labelled in vitro culture supernatants and parasites were collected. The culture supernatants were separated from the parasite pellet by centrifugation at $2,500 \mathrm{~g}$ for five minutes. The supernatants were then centrifugated at $15,000 \mathrm{~g}$ for $20 \mathrm{~min}$ and filtered through a diam. $0.2 \mu \mathrm{m}$ membrane (Acrodisc, Gelman Science). The parasitic pellets and the supernatants were stored at $-80^{\circ} \mathrm{C}$ until used. The same day (day 1), ${ }^{35}$ S]methionine was added to a second flask of in vitro culture of promastigotes and amastigotes; after 24 hours labelling (day 2), the supernatants and the parasites were collected as described above. This procedure was repeated every day during the complete in vitro culture life cycle (five days for amastigote forms and seven days for promastigotes forms). Thus, culture supernatants and parasites were each collected one day after labelling.

\section{IMMUNOPRECIPITATION ASSAYS}

The radiolabelled cells were extensively washed by centrifugation at $2,500 \mathrm{~g}$ in culture medium without 
serum and then incubated in lysis buffer ( $2 \%$ Triton $\mathrm{X}-100,0.6 \mathrm{M} \mathrm{KCl}, 5 \mathrm{mM}$ EDTA (ethylenediamine-tetraacetic acid), $3 \mathrm{mM}$ phenylmethylsulfonyl fluoride, $1 \%$ aprotinin, $2.5 \%$ iodoacetamide in Tris-buffered saline [TBS: $50 \mathrm{mM}$ Tris, $100 \mathrm{mM} \mathrm{NaCl}, \mathrm{pH}$ 7.8]). The lysate was kept on ice for one hour and then centrifuged at $15,000 \mathrm{~g}$ for $10 \mathrm{~min}$ at $4^{\circ} \mathrm{C}$. The Triton X-100 insoluble fraction was discarded and the supernatant (radiolabelled $L$. infantum lysate) was used immediatly or kept at $-80^{\circ} \mathrm{C}$. The radiolabelled supernatants were concentrated by lyophilization.

The radiolabelled $L$. infantum lysate $\left(10^{6} \mathrm{cpm}\right)$ or $100 \mu \mathrm{l}$ of concentrated $L$. infantum supernatant $(2 \mathrm{ml}$ equivalent of labelled supernatant) were each mixed with $2 \mu \mathrm{l}$ of human or dog immune serum and incubated overnight at $4^{\circ} \mathrm{C}$. Then, the antigen-antibody complexes were precipitated by adding $75 \mu \mathrm{l}$ protein A-Sepharose CL4B beads (Pharmacia; Uppsala, Sweden) diluted 1: 1 in TBS, $\mathrm{pH}$ 7.2, with constant stirring at $37^{\circ} \mathrm{C}$ for one hour. After incubation, the complexes were washed: twice with TEN buffer $1(20 \mathrm{mM}$ Tris, 5 mM EDTA, $100 \mathrm{mM} \mathrm{NaCl}$, pH 7.5) supplemented with $1 \% \mathrm{NP}-40$ and $2.5 \%$ bovine serum albumin, four times with TEN buffer 2 (20 mM Tris, $5 \mathrm{mM}$ EDTA, $2 \mathrm{M} \mathrm{NaCl}, \mathrm{pH} 7.5)$ containing $1 \% \mathrm{NP}-40$ and twice with TEN buffer 1 containing $1 \%$ NP-40. The excess buffer was removed, and the immunoprecipitated antigens were prepared for SDS-PAGE and run on $12.5 \%$ polyacrylamide gels under reducing conditions (Laemmli,
1970). The gels were dried, treated by immersion in Amplify (Amersham, France), and exposed to X-OMAT XAR-5 films (Eastman Kodak Co., Rochester, N.Y.). The molecular mass standards $\left({ }^{14} \mathrm{C}\right.$-methylated protein marker) were obtained from Amersham.

\section{RESULTS}

\section{ONE CYCLE L. INFANTUM PROMASTIGOTE ANTIGENS} RECOGNIZED BY HUMAN AND CANINE IMMUNE SERA

1 ive human sera (Fig. 1A) and four canine sera (Fig. 1B) have been respectively used to immunoprecipitate one cycle promastigote antigens. When the promastigote antigens were immunoprecipitated by the different VL-infected human sera (Fig. 1A, lanes 2-6), numerous common bands were found. The major antigens corresponded to molecular weights of 155, 120-118 doublet, 97, 78, 72, 67, 63, 50, 46, 44, 38, 34 and $26 \mathrm{kDa}$. Nevertheless, HIM-C1 (Fig. 1A, lane 4) and HIM-V1 (Fig. 1A, lane 5) sera strongly immunoprecipitated a $63 \mathrm{kDa}$ antigen. When the same antigens were immunoprecipitated by VL-infected canine sera (Fig. 1B, lanes 2-5), the major common bands were $72,70,50,48,46,34-32$ doublet and 22 $\mathrm{kDa}$. Nevertheless, on a general point of view, there were numerous qualitative and/or quantitative differences between $L$. infantum promastigote antigens

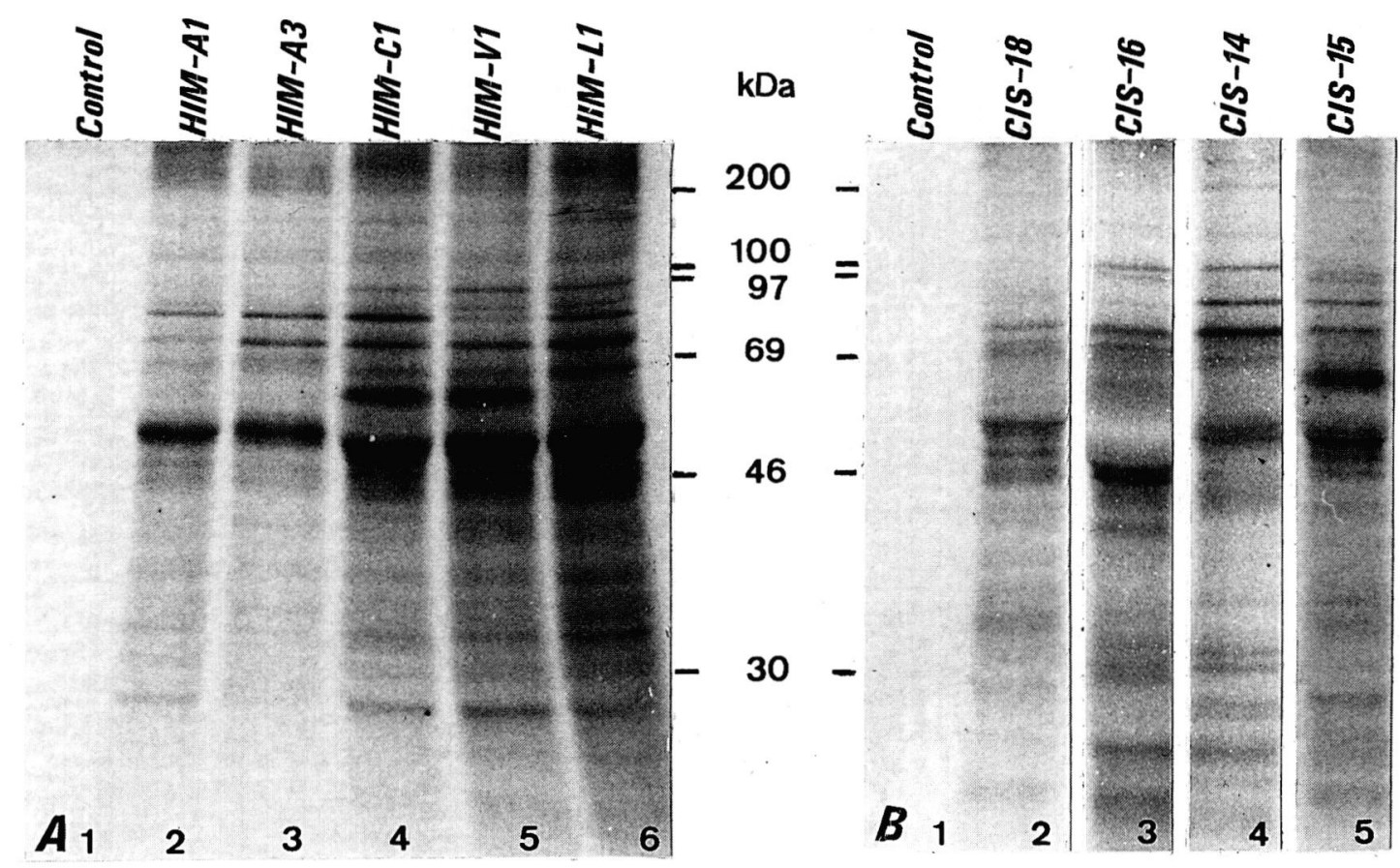

Fig. 1. - Immunoprecipitation of one cycle antigens of L. infantum promastigotes with VL-infected sera. (A) VL-infected human sera: lane 1, healthy human serum; lane 2, HIM-A1; lane 3, HIM-A3; lane 4, HIM-C1; lane 5, HIM-V1 and lane 6, HIM-L1. (B) VL-infected canine sera: lane 1, healthy dog serum; lane 2, CIS-18; lane 3, CIS-16; lane 4, CIS-14 and lane 5, CIS-15. 
immunoprecipited by each canine serum. For example, serum CIS-15 (Fig. 1B, lane 5) stongly reacted with a $63 \mathrm{kDa}$ antigen.

\section{ONE CYCLE L. INFANTUM AMASTIGOTE ANTIGENS RECOGNIZED BY HUMAN AND CANINE IMMUNE SERA}

Similarly, the human sera (Fig. 2A) and canine sera (Fig. 2B) have been used to immunoprecipitate one cycle amastigote antigens. Eleven major common bands, corresponding to proteins of $155,96,78,72$, $66,50,46,38,34,32$ and $26 \mathrm{kDa}$ were immunoprecipitated with both human and canine sera. As demonstrated on the one cycle L. infantum promastigote antigens, HIM-V1 (Fig. 2A, lane 5) exhibited the strongest humoral responses against the one cycle $L$. infantum amastigote antigens. Based on the results obtained with the immunoprecipitations of one cycle L. infantum antigens, HIM-V1 human serum and CIS-15 canine serum were chosen to immunoprecipitate excretedsecreted antigens of $L$. infantum promastigote and amastigote forms.

\section{PromaSTIGOTE EXCRETED-SECRETED ANTIGENS RECOGNIZED BY HUMAN AND CANINE IMMUNE SERA}

The labelled excreted-secreted antigens collected every day of the in vitro life cycle (from day 1 to day 7) were immunoprecipitated with human (HIM-V1) and canine
(CIS-15) serum (Figs. 3A and 3B). All along the in vitro L. infantum life cycle, the major promastigote excretedsecreted antigens (PMES-antigens) inducing humoral response in human were a $63-65 \mathrm{kDa}$ doublet. On day 1 , the major immunoprecipitated antigen had a molecular weight of $63 \mathrm{kDa}$ and three other minor antigens could be detected (78, 70 and $65 \mathrm{kDa}$ ). The excretion of the $65 \mathrm{kDa}$ antigen increased to reach a maximum at day 4 of culture, whereas, the excretion of the 78 and $70 \mathrm{kDa}$ antigens was quantitatively stable until day 4. On day 4, the PMES-antigens that could be immunoprecipitated were 95, 78, 70, 63-65 doublet, 50 and $46 \mathrm{kDa}$. On day 5 to 7 , the patterns of immunoprecipitated antigens looked like those evidenced on day 1 to 3, excepted that there was no qualitative difference between the antigens of 63 and $65 \mathrm{kDa}$.

The immunoprecipitations carried out with canine serum on the same labelled antigens, confirmed the timing of antigen excretion-secretion described above, i.e, an increase from day 1 to day 3, a maximum on day 4 and decrease from day 5 to 7 of culture. Nevertheless, CIS-15 canine serum was able to immunoprecipitate more excreted-secreted antigens than HIM-V1 human serum. The major antigens (detected on day 4) displayed a molecular mass of 120, 78, 74, 72, 65, 63, $50,44,40,38$ and $32 \mathrm{kDa}$. These results showed that the day 4 of in vitro culture (logarithmic growth phase) corresponded, quantitatively, to the most important

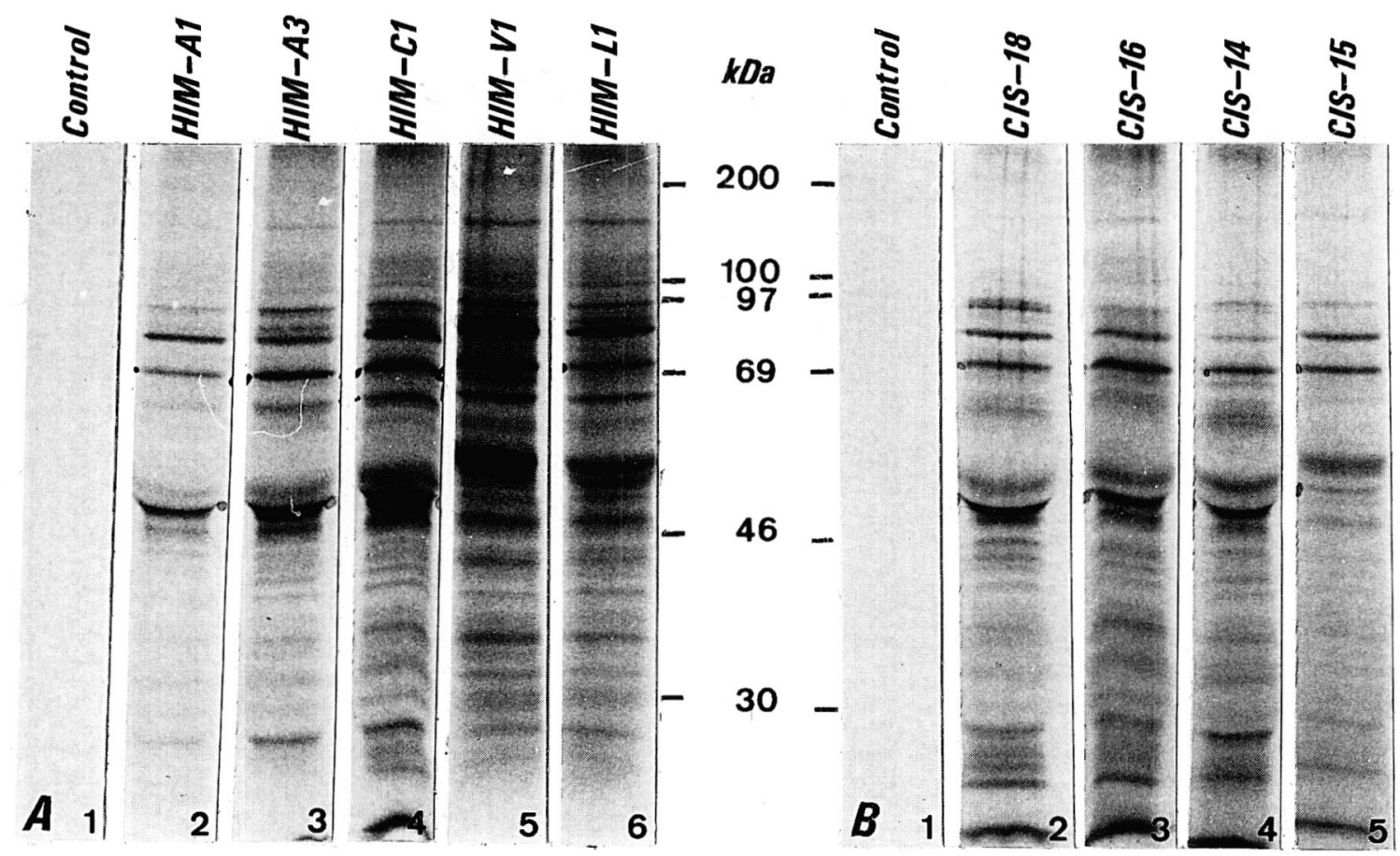

Fig. 2. - Immunoprecipitation of one cycle antigens of L. infantum amastigotes with VL-infected sera. (A) VL-infected human sera : lane 1, healthy human serum; lane 2, HIM-A1; lane 3, HIM-A3; lane 4, HIM-C1; lane 5, HIM-V1 and lane 6, HIM-L1. (B) VL-infected canine sera : lane 1, healthy dog serum; lane 2, CIS-18; lane 3, CIS-16; lane 4, CIS-14 and lane 5, CIS-15. 


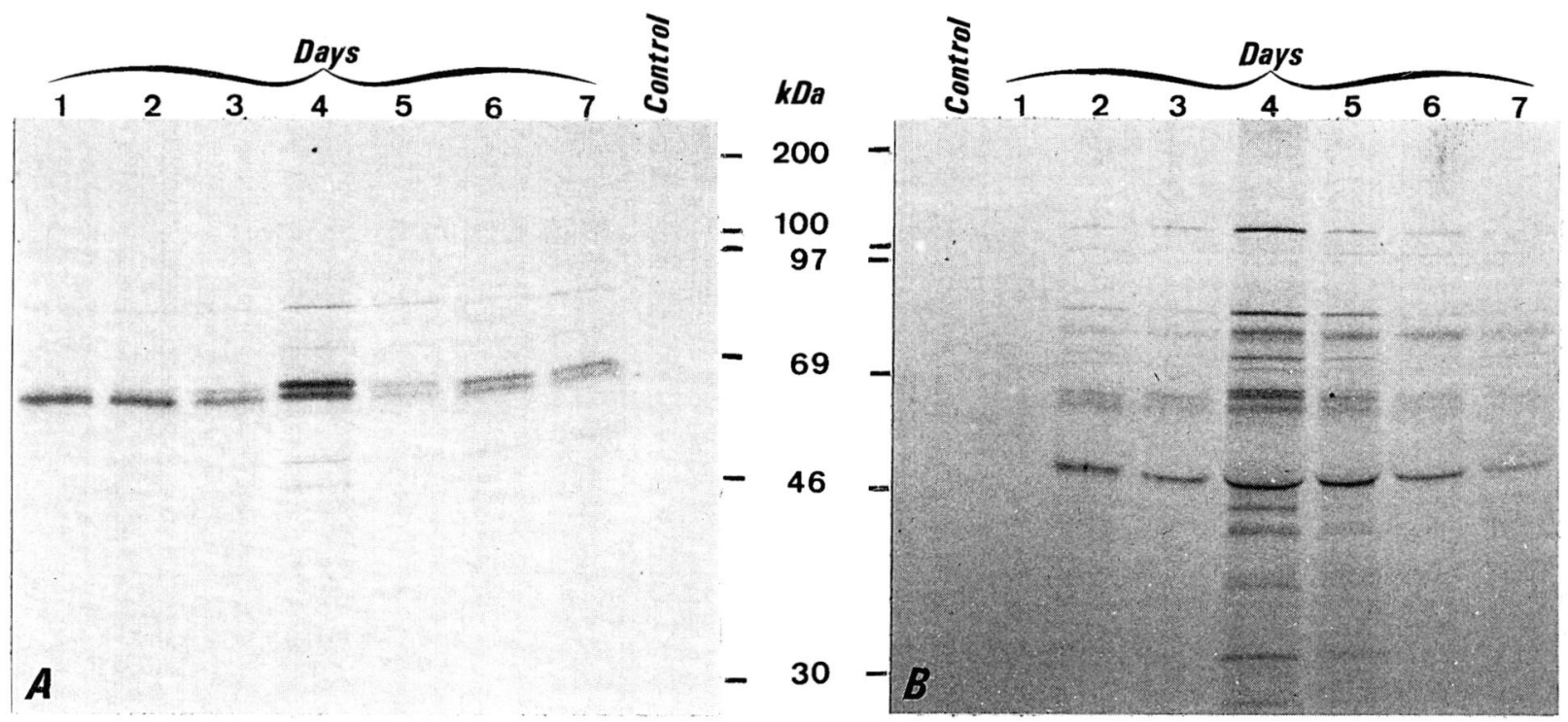

Fig. 3. - Analysis of the humoral response of $\operatorname{man}(\mathbf{A})$ and $\operatorname{dog}(\mathbf{B})$ against promastigote excreted-secreted antigens. Immunoprecipitations were performed with HIM-V1 and CIS-15 sera on radiolabelled culture supernatants collected every day of the in vitro life cycle. Lanes 1 to 7 correspond to each day of the in vitro culture life cycle.

period for $L$. infantum promastigote antigen excretionsecretion.

The human humoral response against PMES-antigens was predominantly directed against a $63-65 \mathrm{kDa}$ excreted-secreted antigen doublet, whereas the canine humoral response was more complex and directed against a greater number of excreted-secreted antigens of L. infantum promastigotes.

\section{AMASTIGOTE EXCRETED-SECRETED ANTIGENS RECOGNIZED BY HUMAN AND CANINE IMMUNE SERA}

The same immunoprecipitation protocol was applied on radiolabelled amastigote excreted-secreted antigens (AMES-antigens) collected from day 1 to day 5 of in vitro culture (Figs. $4 \mathrm{~A}$ and $4 \mathrm{~B}$ ). In contrast to PMESantigens, a similar pattern was observed with human (Fig. 4A) and canine serum (fig. 4B). The major AMESantigens inducing a humoral response had a molecular mass of 90, 67, 65 and $63 \mathrm{kDa}$. However, other minor antigens $(98,81,72,46$ and $44 \mathrm{kDa})$ were also detected. The maximum of excretion-secretion antigens was observed on day 2 to 3 (logarithmic growth phase) of axenic in vitro cultures of $L$. infantum amastigotes. The chronological appearance of the antigens showed that on day 1 (latent growth phase), the $67 \mathrm{kDa}$ antigen was the major secreted antigen. From the beginning of the logarithmic growth phase (day 2), to the end of the logarithmic growth phase (day 4), a strong secretion-excretion of the $67 \mathrm{kDa}$ antigen was observed and an increase in the excretion of the 65 and $63 \mathrm{kDa}$ antigens was evidenced. During the same time lapse (from day 2 to 4 ), there was a qualitative decrease of all the others immunoprecipitated antigens. During the stationary growth phase (day 5), the secretion-excretion of the $67 \mathrm{kDa}$ antigen was residual and all the others excreted-secreted antigens had almost disappeared.

\section{DISCUSSION}

The excreted-secreted antigens of Leishmania are implicated to have a role all along the life cycle 1 of the parasite. In the sandfly vector, at least three enzymes are known to be secreted by promastigotes: chitinase, $\mathrm{N}$-acetylglucasaminidase and sucrase. Chitinase and $\mathrm{N}$-acetylglucasaminidase intervene in the lysis of the chitin framework of the peritrophic membrane which permits the forward migration of the promastigotes (Schlein et al., 1991), the secreted sucrase plays an important role in the nutrition and development of promastigotes in the insect gut (Blum \& Opperdoes, 1994). Early after the infection of the mammalian host, lipophosphoglycan released by the promastigotes, promotes intracellular survival by protecting Leishmania promastigote against early intralysosomal degradation (Mauël, 1996) and on a general point of view, the excreted-secreted antigens of the promastigote stages have also been implicated in facilitating macrophage infection by the parasite (Mukerji et al., 1986), and in decreasing monocyte and lymphocyte differentiation (El-On et al., 1980; Londner et al., 1983). More recently, Peters et al. (1997), have shown that the secretion of proteophosphoglycan by 


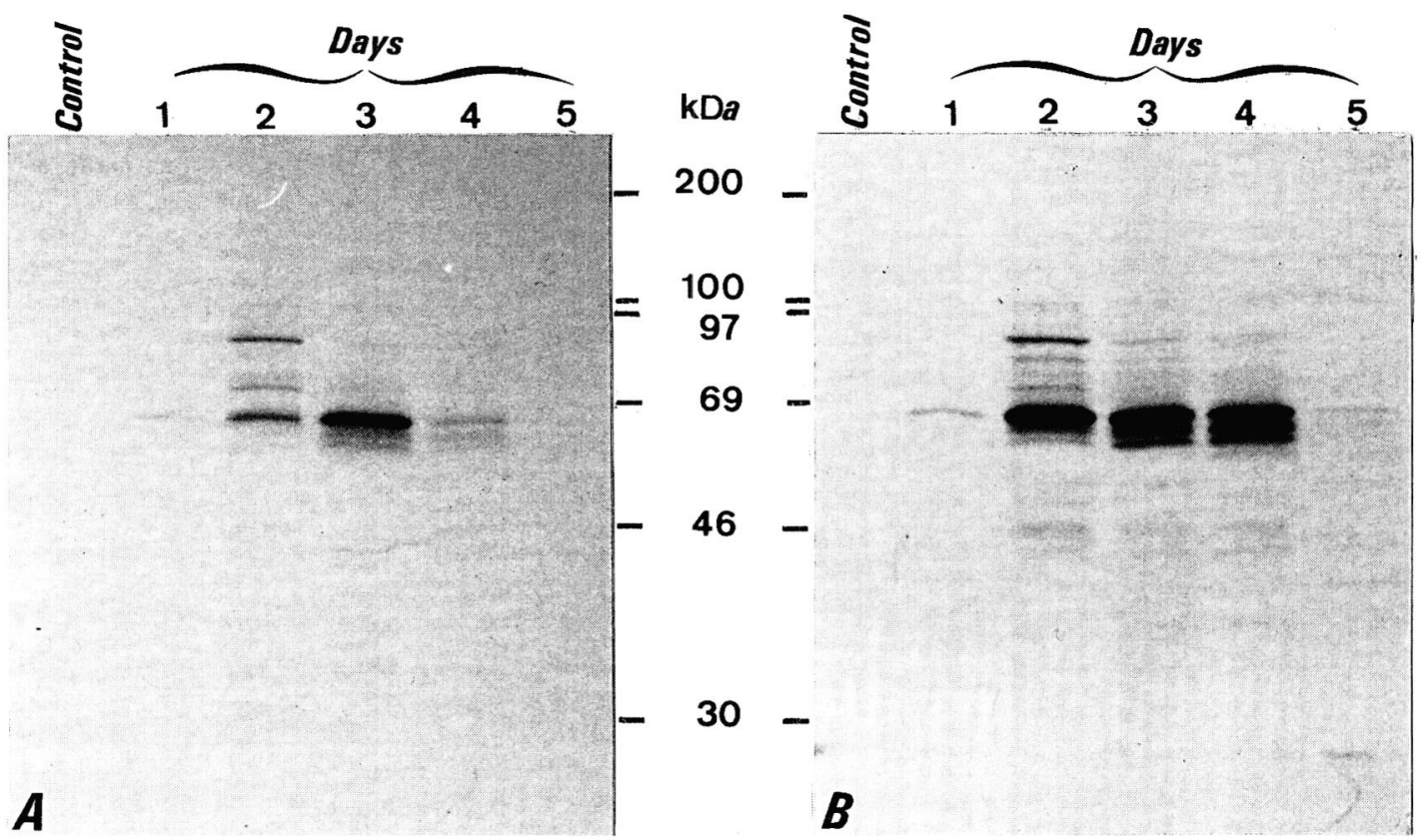

Fig. 4. - Analysis of the humoral response of man (A) and dog (B) against amastigote excreted-secreted antigens. Immunoprecipitations were performed with HIM-V1 and CIS-15 sera on radiolabelled culture supernatants collected every day of the in vitro life cycle. Lanes 1 to 5 correspond to each day of the in vitro culture life cycle.

L. mexicana amastigotes caused vacuolization of peritoneal macrophages in vitro.

In an attempt to define molecules for immunodiagnostic or vaccination purposes, several authors have studied the humoral response to Leishmania promastigote antigens by using immunoblotting techniques with sera from patients affected with leishmaniasis (Rolland-Burger et al., 1991; Mary et al., 1992), and patients cured of visceral leishmaniasis or with an asymptomatic infection (White et al., 1992). However, as far as we know, no studies have been carried out on the L. infantum excreted-secreted antigens, which provide evidence about the humoral immune responses in patients and dogs having visceral leishmaniasis. Our studies illustrate that, regardless the host serum utilized, the excreted-secreted antigens of the promastigote forms or of the intramacrophagic amastigotes are able to induce an humoral response.

The major PMES-antigens able to induce a strong humoral response in man have an apparent molecular weight of 63 and $65 \mathrm{kDa}$. We have observed that in the promastigote stage of $L$. infantum, the $65 \mathrm{kDa}$ excreted-secreted antigen is first, though slightly, secreted during the latent phase, while the secretion strongly increases on day 4 of the in vitro culture (log phase). During the metacyclogenesis phase (days 6 to 7) the excretion of this excreted-secreted antigen remains predominant. In contrast to the $65 \mathrm{kDa}$ antigen, the $63 \mathrm{kDa}$ antigen is more abundantly expressed at the beginning than at the end of the in vitro culture. Thus, the immunoprecipitation assays seem to demonstrate two different roles, for the $63 \mathrm{kDa}$ and $65 \mathrm{kDa}$ excreted-secreted antigens, during the promastigote life cycle. Differences in the expression of these two excreted-secreted antigens of $L$. infantum promastigotes might have a meaning in terms of parasite virulence and/or infectivity. The $63 \mathrm{kDa}$ antigen could be implicated in the establishment of the parasitaemia, whereas the $65 \mathrm{kDa}$ antigen could be involved in the differentiation of promastigote forms to metacyclic forms. The latter hypotheses are in agreement with the studies of Kweider et al. (1989) who have demonstrated that the development of metacyclic promastigotes of $L$. braziliensis is associated with the increasing expression of gp65.

Immunoprecipitations of $L$. infantum promastigotes excreted-secreted antigens with canine infection serum has shown a humoral response directed against a greater number of antigens than that with human serum. In addition to the $63 \mathrm{kDa}$ and $65 \mathrm{kDa}$ excretedsecreted antigens majoritarely recognized by human serum, the canine serum allowed the detection of antigens in the $70-120 \mathrm{kDa}$ range and in the low molecular mass range. Our data demonstrate that the promastigotes predominantly release excreted-secreted antigens on day 4 , during the log phase of the in vitro culture. This stronger humoral response of dogs against leishmanial excreted-secreted antigens is in accordance 
with the studies of different authors (Abranches et al., 1991; Rolland et al., 1994; Correia et al., 1996). In this case, the authors studied the humoral response of infected dogs against total antigenic extracts of the promastigote forms and, by western blotting, they have shown that the strongest humoral response was directed against the $30,45 \mathrm{kDa}$, the $58-84 \mathrm{kDa}$ and the $94 \mathrm{kDa}$ regions. If we compare the human and the canine humoral responses, the hypothesis that the canine response could be directed against antigens of lyzed promastigotes must be discarded. As a matter of fact, if the immunoprecipitation samples had contained mainly lysis antigens, the immunoprecipitation patterns of promastigote excreted-secreted antigens with human or canine sera should have been similar to that of the immunoprecipitation patterns observed when using the total promastigote antigens. Indeed, we can observe that the human antibody response is completely different when using total or excreted-secreted antigens.

The success of having axenically grown amastigotes with biochemical and molecular characteristics similar to those of macrophage-derived amastigotes has allowed new developments in the studies of excretedsecreted antigens from the amastigote stage. In contrast to the data obtained with the immunoprecipitations on the excreted-secreted antigens of the promastigote form of $L$. infantum, the pattern of immunoprecipitated amastigote excreted-secreted antigens and their secretion timing were quite comparable when human or canine sera were used. Working with axenically grown amastigotes implicates that at least three different types of excreted-secreted antigens may be observed. The excreted-secreted antigens present in/on the parasitophorous vacuole, in/on the macrophage, and outside the host-cell. Even if the results obtained do not allow for discrimination between these different excretedsecreted antigens, it can be assumed that the excretedsecreted antigens immunoprecipitated during the first two days of in vitro culture could be directly involved in the parasite establishment inside the parasitophorous vacuole. Lang et al. (1994) have suggested that the parasitophorous vacuole acquires its biochemical characteristics within 48 hours after infection. In accordance with these results, it is demonstrated here that the immunoprecipitation profile of the excreted-secreted antigens was qualitatively complex on day 2 and remained more simple on days 3 and 4 . In addition, Lang et al. (1994) have shown that a GTP-binding protein rab (marker of the prelysosomal compartment) was highly expressed in/on the parasitophorous vacuole. In a similar manner, when polyclonal antibodies directed against excreted-secreted antigens, were used to screen a cDNA library of $L$ infantum amastigote stages, among the different cDNA clones obtained, one of them expressed an excreted-secreted GTP-binding protein rab (unpublished results).

With regard to the excretion timing of the amastigote excreted-secreted antigens during the in vitro life cycle, a strong humoral response against a 67,65 and $63 \mathrm{kDa}$ triplet was observed from day 2 to day 4 of culture. A polyclonal antibody directed against the major surface glycoprotein gp63 is able to recognize these excretedsecreted antigens (data not shown). Different authors [Medina-Acosta et al. (1989, 1993), Frommel et al. (1990)] have demonstrated that the gp63 can be found in the amastigote stage. The amastigote gp63, unlike the promastigote form of the protein, lacks a phosphatidyl inositol membrane anchor and is an hydrophilic protein that can be found in the flagellar pocket of the parasite. Moreover, Frommel et al. (1990) demonstrated that at least two higher-Mr gp63 bands can be detected in an amastigote lysate. Medina-Acosta et al. (1993) have shown that the gp63 genes expressed in the amastigote encode an extended ( 41 amino acids) carboxyl terminus. In addition, Frommel et al. (1990) have also supposed that these differences in the molecular weigth possibly reflected differences in the posttranslational processing of the amastigote gp63. In this experiment, it can be observed that, although the 67 $\mathrm{kDa}$ antigen was expressed from day 2 to day 4 , the 65 and $63 \mathrm{kDa}$ excreted-secreted antigens were only expressed on days 3 and 4 and could not be immunoprecipitated on day 2 . That seems to indicate that these different gp63 proteins and/or post-translational modifications probably possess a biological significance for the development of the parasite inside the host-cell. In conclusion, we demonstrated the release of extracellular antigens from L. infantum amastigote and promastigote forms and the existence of a host humoral response directed against these excreted-secreted antigens. Moreover, the time course studies have shown that the secretion of leishmanial antigens exhibits both qualitative and quantitative variations according to the day of the in vitro cycle. Currently, studies are underway to demonstrate the efficiency of excretedexcreted antigens to induce an immunoprotection against $L$. infantum. Moreover, the biochemical characterization of excreted-secreted antigens will allow us to speculate about their possible functions in the survival of amastigotes within the macrophage.

\section{REFERENCES}

Abranches P., Santos Gomes G., Campino L., Schnur L.F. \& JAFFE C.L. An experimental model for canine visceral leishmaniasis. Parasite Immunology, 1991, 13, 537-550.

Alexander J. \& Russel D.G. The interaction of Leishmania species with macrophages. Advances in Parasitology, 1992, 31, 175-254. 
Bates P.A., Hermes I. \& Dwyer D.M. Leishmania donovani: immunochemical localization and secretory mechanism of soluble acid phosphatase. Experimental Parasitology, 1989, $68,335-346$.

Bates P.A., Hermes I. \& Dwyer D.M. Golgi-mediated posttranslational processing of secretory acid phosphatase by Leishmania donovani promastigotes. Molecular and Biochemical Parasitology, 1990, 39, 247-255.

Bates P.A., Robertson C.D., Tetley L. \& Coombs G.H. Axenic cultivation and characterization of Leishmania mexicana amastigote-like forms. Parasitology, 1992, 105, 193-202.

Berman J. \& Dwyer D.M. Expression of Leishmania antigen on the surface membrane of infected human macrophages in vitro. Clinical of Experimental Immunology, 1981, 44, 342-348.

Blum J.J. \& Opperdoes F.R. Secretion of sucrase by Leishmania donovani. Journal of Eukaryotic Microbiology, 1994, 41, 228-231.

Carvalho E.M., Teixeira R.S. \& Johnson W.D. Cell-mediated immunity in American visceral leishmaniasis: reversible immunosupression during acute infection. Infection and Immunity, 1981, 33, 498-500.

Chakraborty P. \& Basu M.K. Leishmania phagolysosome: drug trafficking and protein sorting across the compartment. Critical Review of Microbiology, 1997, 23, 253-268.

Correia da Costa J.M., Neocy Asit B., Vouldoukis I., Silva Sampaio M.L., Gentilini M. \& Monjour L. Antigenic components of partially purified antigens of Leishmania donovani infantum recognized by sera from dogs with asymptomatic or active visceral leishmaniasis. American Journal of Tropical Medicine and Hygiene, 1996, 55, 511-515.

DOYLE P.S. \& DWyer D.M. Leishmania: immunochemical comparison of the secretory (extracellular) acid phosphatases from various species. Experimental Parasitology, 1993, 77, 435-444.

El-On J., Bradley D.J. \& Freeman J.C. Leishmania donovani: action of excreted factors on hydrolytic enzyme activity of macrophages from mice with genetically resistance to infection. Experimental Parasitology, 1980, 49, 167-174.

Frommel T.O., Button L.L., Fujikura Y. \& McMaster W.R. The major surface glycoprotein (GP63) is present in both life stages of Leishmania. Molecular and Biochemical Parasitology, 1990, 38, 25-32.

GalvaO-Castro B., Sa Ferreira J.A., Marzochi K.F., Marzochi M.C., Coutinho S.G. \& Lambert P.H. Polyclonal B cell activation, circulating immune complexes and autoimmunity in human american visceral leishmaniasis. Clinical and Experimental Immunology, 1984, 56, 58-66.

Handman E., Jarvis H.M. \& Mitchell G.M. Leishmania major, identification of stage-specific antigens and antigens shared by promastigotes and amastigotes. Parasite Immunology, $1984,6,223-233$.

Ilg T., Stierhof Y.D., Etges R., Adrian M., Harbecke D. \& OveRATH P. Secreted acid phosphatase of Leishmania mexicana: a filamentous phosphoglycoprotein polymer. Proceedings of the National Academy of Science USA, 1991, 88, 8774-8778.
Ilg T., Stierhof Y.D., McConville M.J. \& Overath P. Purification, partial characterization and immunolocalization of a proteophosphoglycan secreted by Leishmania mexicana amastigote. European Journal of Cell Biology, 1995, 66, 205-215.

Lang T., Hellio R., Kaye P.M. \& Antoine J.C. Leishmania donovani-infected macrophages: characterization of the parasitophorous vacuole and potential role of this organelle in antigen presentation. Journal of Cell Science, 1994, 107, 2137-2150.

King D.L., Chang Y.D. \& Turco S.J. Cell surface lipophosphoglycan of Leishmania donovani. Molecular and Biochemical Parasitology, 1987, 24, 47-53.

Kweider M., Lemesre J.L., Santoro F., Kusnierz J.P., SAdiGURSKY M. \& CAPRON A. Development of metacyclic Leishmania promastigotes is associated with the increasing expression of GP65, the major surface antigen. Parasite Immunology, 1989, 11, 197-209.

Laemmli U.K. Cleavage of structural proteins during the assembly of the head of bacteriophage T4. Nature, 1970, 227, 680-685.

Lemesre J.L., Sereno D., Daulouede S., Veyret B., Brayon N. \& Vincendeau P. Leishmania spp.: nitric oxide-mediated metabolic inhibition of promastigote and axenically grown amastigote forms. Experimental Parasitology, 1997, 86, 5868 .

Londner M.V., Frankenburg Slutzky G.M. \& Greenblatt C.L. Action of leishmanial excreted factors (EF) on human lymphocyte blast transformation. Parasite Immunology, 1983, 5, 249-256.

MAUËL J. Intracellular survival of protozoan parasites with special reference to Leishmania spp., Toxoplasma gondii and Trypanosoma cruzi. Advances in Parasitology, 1996, 38, $1-51$.

Mary C., Lamouroux D., Dunan S. \& Quilici M. Western blot analysis of antibodies to Leishmania infantum antigens: potential of the $14 \mathrm{KD}$ and the $16 \mathrm{KD}$ antigens for diagnostis and epidemiologic purposes. American Journal of Tropical Medecine and Hygiene, 1992, 47, 764-771.

Medina-Acosta E., Karess R.E., Schwartz H. \& Russell. D.G. The promastigote surface protease (gp63) of Leishmania is expressed but differentially processed and localized in the amastigote stage. Molecular and Biochemical Parasitology, 1989, 37, 263-274.

Medina-Acosta E., Karess R.E. \& Russell D.G. Structurally distinct genes for the surface protease of Leishmania mexicana are developmentally regulated. Molecular and Biochemical Parasitology, 1993, 57, 31-46.

Mukerji K., Das A.K., Banerjee N. \& Ghosh D.K. Studies on exoantigens of Leishmania donovani. Indian Journal of Biochemistry and Biophysics, 1986, 23, 148-151.

Prina E., Lang T., Glaichenhaus N. \& Antoine J.C. Presentation of the protective parasite antigen LACK by Leishmania-infected macrophages. Journal of Immunology, 1996, 156, 4318-4327.

Peters C., Stierhof Y.D. \& Ilg T. Proteophosphoglycan secreted by Leishmania mexicana amastigote causes vacuole formation in macrophages. Infection and Immunity, 1997, 65, 783-786. 
Rolland-Burger L., Rolland X., Grieve C.W. \& Monjour L. Immunoblot analysis of the humoral immune response to Leishmania donovani infantum polypeptides in human visceral leishmaniasis. Journal of Clinical Microbiology, 1991, 29, 1429-1435.

Rolland L., Zilberfarb V., Furtado A \& Gentilini M. Identification of a 94-kilodalton antigen on Leishmania promastigote forms and its specific recognition in human and canine visceral leishmaniansis. Parasite Immunology, 1994, 16, 599-608.

Schlein Y., Jacobson R.L. \& Shlomai J. Chitinase secreted by Leishmania functions in sandfly vector. Proceedings of the Royal Society of London, 1991, 245, 121-126.

Turco S.J. \& Descoteaux A. The lipophosphoglycan of Leishmania parasites. Annual Review of Microbiology, 1992, 46, 65-94.

White A.C., Caste M., Garcia L., Trujlllo D. \& Zambrano L. Leishmania chagasi antigens recognized in cured visceral . leishmaniasis and asymptomatic infection. American Journal of Tropical Medecine and Hygiene, 1992, 46, 123131.

Williams K.M., Sacci J.B. \& Anthony R.L. Identification and recovery of Leishmania antigen displayed on the surface membrane of mouse peritoneal macrophages infected in vitro. Journal of Immunology, 1986, 136, 1853-1858.

Yahiaoui B., Loyens M., Taibi A., Schöneck R., Dubremetz J.F. \& OuAissi M.A.. Characterization of a Leishmania antigen associated with cytoplasmic vesicles ressembling endosomal-like structure. Parasitology, 1993, 107, 497-507.

Reçu le 15 décembre 1998 Accepté le 26 mars 1999 